\title{
LA SOCIEDAD DEL RIESGO Y LA NECESIDAD MODERNA DE SEGURIDAD
}

\section{Antonio Martín Cabello Jaime Hormigos Ruiz*}

\section{RESUMEN}

Este artículo trata de mostrar el desarrollo de una sociedad del riesgo mundial. El proceso de globalización lleva a una interconexión mayor de los riesgos locales. En consecuencia, un nuevo concepto de seguridad está surgiendo fuertemente relacionado con estos cambios. El artículo, finalmente, muestra las implicaciones de la sociología del riesgo en la construcción de la subjetividad humana.

\section{PALABRAS CLAVE}

Riesgo. Sociedad del Riesgo. Inseguridad. Vulnerabilidad. Post-modernidad.

\section{ABSTRACT}

This paper tries to show the development of a world risk-society. The globalisation process leads to a higher interconnection of local risks. Consequently, a new security concept is emerging strongly related with this changes. The paper, finally, shows the implications of risk-society in the construction of human subjectivity.

\section{KEY-WORDS}

Risk. Risk-Society. Insecurity. Vulnerability. Post-modernity.

\section{INTRODUCCIÓN}

La seguridad es un concepto de vital importancia dentro de las sociedades contemporáneas. Todos los modelos de sociedad han desarrollado funciones de

\footnotetext{
*Profesores de sociología de la Universidad Rey Juan Carlos y miembros de la ACMS.
} 
seguridad y control, pero éstas nunca alcanzaron las dimensiones que están tomando hoy en día. El campo de acción de la seguridad se ha vuelto tan amplio que posee una naturaleza pluridimensional, conectada con instituciones jurídicas, políticas, económicas, policiales o asistenciales; y conforma el orden que demanda un nuevo tipo de sociedad donde el conflicto se presenta como algo natural dentro del proceso de interacción. Estamos enfrentados a continuas transformaciones en las cuales se plantean formas de riesgo que se apartan de las existentes en épocas pasadas. Actualmente, los riesgos que debemos asumir tienen un origen incierto y sus consecuencias no pueden determinarse. Aparecen nuevas opciones y peligros para la vida en sociedad que demandan una nueva estructura para gestionar el conflicto, pues nos vemos obligados a responder constantemente a nuevas amenazas y a una pérdida en el nivel de seguridad demandado por todos. Se impone un nuevo modelo de seguridad capaz de enfrentar peligros no limitados ni espacial, ni temporal o socialmente. Este modelo debe adaptarse a profundos cambios en la sociedad, para así poder ofrecer soluciones concretas a los problemas sociales actuales.

El intento de gestionar el riesgo es la consecuencia lógica de la llamada "sociedad del riesgo global". La modernidad implica un ser humano cada vez más racional, que intenta controlar todas las facetas de su mundo vivencial. Las ideologías del progreso o del bienestar colectivo son consecuencias lógicas de este deseo. Así, no es extraño que la falta de dominio, tanto de las acciones como de sus consecuencias sean estas intencionales o no, genere ansiedad y frustración social. Los debates actuales en los que se contrapone libertad y seguridad descansan, en buena medida, en este deseo de seguridad y en la frustración por la falta de control sobre un mundo social e individual cada vez más imprevisible.

El artículo plantea una de las consecuencias más importantes de la globalización: el nacimiento de una sociedad del riesgo global. Describe esta aparición en cuatro partes. En primer lugar, el desarrollo de una sociedad global y de un nuevo modo de conceptuar la seguridad. La creciente interconexión no sólo mundializa los flujos financieros, sino toda una serie de riesgos. Así, en segundo lugar, se plantea un nuevo medioambiente de inseguridad colectiva global y la ineficacia de los mecanismos tradicionales de control del riesgo. El resultado de esa nueva situación mundial es apuntado en tercer lugar, describiendo la concepción del riesgo propia de las sociedades tradicionales, modernas y de las actuales postmodernas. Por último, se hace hincapié en las consecuencias para la subjetividad humana de este nuevo entorno de riesgos.

\section{EL DESARROLLO DE LA SOCIEDAD GLOBAL Y DEL CONCEPTO DE SEGURIDAD}

Los acontecimientos desencadenados tras el 11 de septiembre de 2001 no han hecho sino poner de manifiesto la existencia, en nuestras sociedades, de 
tensiones y conflictos que se desarrollan sobre una perspectiva global. En Nueva York, Madrid, Londres, así como en otros lugares del planeta, se ha podido constatar situaciones que responden a la aparición de conflictos, gestados de forma subterránea durante las últimas décadas con la misma rapidez con la que crecían las desigualdades en el mundo. Además de poner de manifiesto la tensión existente entre las distintas formas de percibir el mundo, estos actos de terrorismo nos han mostrado hasta que punto hemos desarrollado una verdadera interdependencia global buscando la unidad del planeta, también en materia de seguridad.

Las sociedades desarrolladas creyeron que fortificando su territorio podrían ser capaces de garantizar la seguridad. Apostaron por blindar todo lo que quedaba dentro de sus fronteras y regular y controlar la entrada de todo aquello que pudiera parecer extraño. Pero, tal vez, los últimos acontecimientos hayan puesto en tela de juicio esta idea demostrando que el lugar ya no protege. Las sociedades más desarrolladas del planeta ven ahora amenazada su seguridad incluso dentro de su espacio. El concepto de seguridad se vuelve difuso, se convierte en un problema extraterritorial. La actual inseguridad global se encuentra diluida en la confrontación entre el espacio y el tiempo. Debido a esto, no existen mecanismos institucionalizados para enfrentar esta inseguridad. Cada acto de violencia conduce a una respuesta distinta que a su vez genera otro conflicto. Además, como el equilibrio de poderes y el espectro de oportunidades oscilan, las tensiones de vital importancia ayer, pasan hoy a un segundo plano para acometer los nuevos frentes de batalla que van surgiendo como consecuencia de la creciente inseguridad global.

Cada conflicto se platea en un espacio determinado y en un tiempo concreto, pero su desarrollo tiene consecuencias para todo el planeta debido a la creciente interconexión global. Intentar atajar estos conflictos aplicando medidas que intenten hacer desaparecer la tensión únicamente en la zona en la que aparece, no hace sino enmascarar el problema. Tratar de detener la inseguridad desde el interior de un territorio, entendiéndola como algo local y utilizando únicamente los medios disponibles en este ámbito, está provocando lo que Zygmunt Bauman denomina una "sobrecarga de seguridad" (2002: 115); que se traduce en una desviación de los problemas y preocupaciones derivados de la inseguridad hacia el terreno de la seguridad activa. En la sociedad actual vemos crecer la vigilancia a través de controles policiales, circuitos cerrados de cámaras, dispositivos electrónicos para evitar robos, etc. Estas medidas activas de seguridad son las únicas con la posibilidad de ser vistas como prueba de que se está haciendo algo, porque no existe una política en el orden mundial con posibilidad de actuar más allá del ámbito de la seguridad activa.

\section{LA INSEGURIDAD COLECTIVA Y EL CONTROL DEL RIESGO}

El riesgo está presente, en mayor o menor medida, en todos los ámbitos de la vida social. No debemos olvidar que todas las sociedades humanas, 
independientemente de su grado de desarrollo son conflictivas y polémicas. Siguiendo a Karl Marx podemos decir que el conflicto es el motor del cambio social, se encuentra situando en la propia estructura de la sociedad. Ralf Dahrendorf consideraba que existían causas endógenas a la propia estructura del sistema que explican el conflicto; y en las sociedades avanzadas esa razón es la desigual distribución de la autoridad. En todas las sociedades se dan una serie de situaciones de oposición o enfrentamiento en torno a unos intereses determinados. Los conflictos generan tensiones que conducen a nuevos cambios sociales. La estructura de la sociedad actual excluye la unanimidad y en este contexto es más raro encontrar consenso que situaciones conflictivas. Consenso y conflicto se alternan dando una nueva dimensión a los riesgos que debemos asumir en la vida social. Esta situación conflictiva se hace más patente con el desarrollo de la sociedad global y la creciente conexión entre sociedades. En este contexto, el conflicto y el riesgo asumen dimensiones mucho más complejas. Hemos aprendido a vivir con los conflictos, hemos aprendido a gestionar las situaciones conflictivas que nos rodean, en nuestras relaciones sociales, laborales, etc., pero hoy es necesario aprender a gestionar los nuevos riesgos que aparecen como consecuencia de la dimensión global. El riesgo se ha convertido en algo natural de la sociedad humana, en parte del proceso de interacción social. El riesgo es hoy pluridimensional, no hay una única causa que explica el riesgo. Tiene una perspectiva global, pero experimentamos sus consecuencias individualmente, dependiendo de nuestras circunstancias sociales.

La misión de los modernos sistemas de seguridad consiste en detectar dónde pueden surgir esos riesgos y ponerles remedio. El problema es que dentro de las sociedades actuales el riesgo es imprevisible. Es el resultado de una serie de factores interconectados, unos fácilmente previsibles y otros imposibles de localizar; ya que cambian al mismo ritmo que lo hace la sociedad. Para evitar riesgos y responder rápidamente a lo imprevisible resulta necesaria una gran centralización organizativa, que demanda una localización de las situaciones de riesgo que permita poner remedio a casos concretos; pero, paradójicamente, en el mundo de la globalización es imposible crear tal estructura porque el riesgo también es global.

Ante esta paradoja rápidamente surgen las pregunta: ¿Cómo es posible atajar los riesgos actuales si es imposible localizarlos? o ¿qué mecanismos de seguridad son necesarios aplicar en estas sociedad del riesgo global? La respuesta es incierta, pues en la sociedad actual parece más fácil definir la inseguridad que la seguridad. La inseguridad parece marcar nuestras vidas. El desarrollo social ha ido creando estructuras donde los intereses individuales priman sobre el grupo, donde el individuo deja de conocer su entorno más cercano porque ha perdido la capacidad de interactuar con él. Vivimos en una ciudad, pero apenas conocemos a nuestros vecinos, trabajamos fuera, pasamos mucho tiempo intentando desarrollar unas relaciones laborales plenas y esto nos hace descuidar las relaciones con nuestro entorno más cercano, que desconocemos y que, en demasiadas ocasiones, se 
presenta ante nosotros como extraño, desconocido. Echamos en falta la seguridad, una cualidad crucial para una vida feliz, pero una cualidad que la sociedad que habitamos es incapaz de ofrecer. De este modo, "nuestra inseguridad aumentan a medida que seguimos adelante" (Bauman, 2003: 169).

En la era de la globalización, las fuentes de la inseguridad ya no son visibles, no se pueden localizar con precisión. Quedamos inmersos en un mundo impredecible de desregularización, flexibilidad, competitividad e incertidumbre endémicas, pero cada uno de nosotros sufre estos cambios por sí mismo, como un problema privado. Tendemos a buscar un remedio para el malestar de la inseguridad en el cuidado de la seguridad, que tratamos de localizar en nuestro entorno más cercano. Intentamos sentirnos seguros en nuestro hogar, blindamos todo cuanto nos rodea con el objetivo de crear un ambiente tranquilo, exento de peligros. Pero, entonces, escondidos en nuestro búnker particular, se convierten en sospechosos todos aquellos que nos rodean, que parecen distintos, extraños y extranjeros, cuyo comportamiento nos resulta imprevisible. "Los extraños son la amenaza encarnada, por lo que personifican vicariamente esa inseguridad que acosa nuestra vida: los temores difusos y dispersos, difíciles de individualizar y denominar, tienen ahora un objetivo tangible en el que centrarse; creemos conocer donde residen los peligros" (Bauman,2003:170).

Buscamos seguridad dentro de nuestro entorno más cercano, que ha cambiado y ahora se nos presenta cada vez más hostil. Demandamos mayor seguridad, pero somos incapaces de hacer frente a todas las dimensiones que el concepto presenta en la actualidad, por lo cual tendemos a centrarnos en los mecanismos de seguridad visible; que, en muchas ocasiones, cumplen su función a costa de nuestra libertad. Entramos así en un nuevo conflicto, debemos ser capaces de elegir entre seguridad o libertad, dos dimensiones que resultan fundamentales para la vida en las sociedades más desarrolladas del planeta. Parece, pues, que no podemos escapar al creciente conflicto entre seguridad y libertad. Únicamente se nos presente la posibilidad de participar en él asumiendo riesgos, evaluando individualmente las oportunidades y peligros que se nos presentan en el día a día.

\section{LA SOCIEDAD DEL RIESGO GLOBAL}

El ser humano siempre ha tratado de prever el futuro, es decir, de adelantar los hechos futuros con el fin de eliminar o disminuir los riesgos y asegurarse el éxito. Para ello, se ha recurrido a toda una serie de estrategias: hacer ofrendas a los dioses, consultar adivinos y pitonisas y, más recientemente, realizar precisos cálculos de probabilidades con ayuda de la ciencia para reducir las posibilidades de error. El modo en que encaramos la incertidumbre y controlamos el riesgo ha ido cambiando a lo largo de la historia. Podemos distinguir tres aproximaciones fundamentales: en la sociedad tradicional, en la moderna y, finalmente, en las actuales sociedades postmodernas. 


\subsection{El riesgo en la sociedad tradicional}

El ser humano en las sociedades tradicionales era ampliamente consciente de los riesgos que suponía la existencia: posibilidad de muerte, enfermedad, padecimientos, etc. Su capacidad de controlar el mundo, sin embargo, era bastante limitada dado su escasa capacidad técnica (el subdesarrollo de la medicina, por ejemplo, que no podía hacer frente a las crisis sanitarias cíclicas como las pestes; o de la técnica agraria, que era incapaz de combatir las limitaciones del tiempo y de la tierra, favoreciendo la aparición de hambrunas periódicas). El ser humano, no obstante, tenía la necesidad de intentar controlar la naturaleza y recurría sobre todo a la magia y la religión para intentar predecir el futuro (adivinos, pitonisas, etc.) e intentar cambiar su rumbo (mediante ofrendas, sacrificios, penitencias, etc.). Este comportamiento y estas creencias producían una actitud ante la vida fatalista, ya que el mal era inevitable si la divinidad no intervenía en el desarrollo de los acontecimientos. El ser humano, sometido a limitaciones derivadas de su escaso control técnico del mundo, luchaba contra las mismas pero no podía sino reconocer su dependencia del destino.

\subsection{El riesgo en la sociedad moderna}

La sociedad moderna supone una ruptura con las nociones tradicionales sobre el riesgo. Los nuevos descubrimientos científicos, la técnica, la medicina, el control de la producción agraria e industrial, el carácter electivo de los gobiernos, etc., impulsaron una nueva concepción sobre el mundo. El riesgo pasó a ser contemplado como algo que la ciencia podría controlar. El futuro de los seres humanos ya no descansaba en causas debidas al azar o el destino, sino que se encontraba en sus manos. El avance de la civilización, pues, iría eliminando el mal. En este sentido, se creía que los males de este mundo irían desapareciendo con el progreso continuo de la humanidad (Bury, 1971; García Morente, 1980; Nisbet, 1980). La modernidad traería la desaparición de los riegos. El mundo dejaría de ser un entorno hostil y se transformaría en un lugar donde el ser humano correría menos riesgos. La gestión de los bienes, el control racional del mundo, haría que los riesgos disminuyeran y que los bienes fueran, cada vez más, patrimonio de toda la humanidad.

Para Max Weber, las sociedades modernas se caracterizan por un continuo proceso de racionalización. Todas las facetas de la vida social se iban haciendo más racionales, es decir, cuantificables, valorables (reducible a medida), previsibles y controlables (Ritzer, 1999). Esto provocaba que dominios mágicos dieran paso a una visión racional de la vida. La salud y la enfermedad, la vida y la muerte no eran ya fenómenos debidos a las malos o buenos hálitos, ni al deseo de una voluble divinidad. Por el contrario, el ser humano era capaz de hallar las causas que movían 
el mundo natural. Esto producía lo que Weber llamó el "desencantamiento" del mundo. La realidad no se explicaba según criterios míticos o mágicos, sino en función de leyes abstractas descubiertas por la ciencia. La consecuencia lógica era que los seres humanos eran capaces de prever el devenir y, en cierta medida, de controlarlo. El binomio ciencia y técnica permitía controlar la realidad mediante el uso de la razón. Este proceso social resultó clave en la configuración del universo moderno, que tan familiar nos resulta. La empresa capitalista, el crecimiento de la vida urbana, los dominios de la política, la religión o el arte no se explican sin hacer referencia a un nuevo modo de afrontar la realidad: el proceso de racionalización.

Los seres humanos se sintieron seguros de su control del mundo. La ciencia y la técnica permitían asegurar la producción de alimentos, vencer enfermedades antaño mortíferas, viajar por todo el mundo en poco tiempo, etc. Tanto fue así que de modo generalizado se comenzó a creer que el mundo cambiaba necesariamente a mejor, que progresaba ineludiblemente. Además, se empezaron a asegurar las posibles eventualidades de la vida, convirtiéndose el azar en riesgo, en una eventualidad calculada. Durante el siglo XIX y la primera mitad del siglo XX se desarrollaron seguros privados y públicos que hacían del mundo un lugar más previsible. El mismo Estado de Bienestar no es sino la expresión máxima de este espíritu de aseguramiento.

El hombre moderno, en consecuencia, era un hombre seguro de sí mismo, de su capacidad técnica para domeñar el mundo gracias a la ciencia y al análisis racional. Si algo fallaba no era sino por una carencia de la ciencia, aún no lo suficientemente desarrollada para controlar ese fenómeno. El fatalismo previo dio paso a un optimismo que consideraba que el mundo avanzaba inexorablemente y que lo hacía a mejor. Y los grandes pensadores de este avanzar eran los médicos e ingenieros, los "industriales" de Saint-Simon y Comte. Los científicos y tecnólogos ocuparon el nicho que los sacerdotes dejaron en el pasado.

\subsection{El riesgo en la sociedad postmoderna}

La actual sociedad postmoderna sería una sociedad en la cual la concepción del riesgo ha variado mucho respecto a la de la sociedad tradicional y moderna. Como afirma el sociólogo Richard Sennet: "A lo largo de la mayor parte de la historia humana, la gente ha aceptado que la vida cambia de repente por culpa de las guerras, las hambrunas y otras catástrofes, y también que, para sobrevivir, hay que improvisar. (...) Lo que hoy tiene de particular la incertidumbre es que existe sin la amenaza de un desastre histórico; y en cambio, está integrada en las prácticas cotidianas de un capitalismo vigoroso" (2005: 30). Tras la Segunda Guerra Mundial se produjeron toda una serie de cambios en la estructura de las sociedades capitalistas, que modificaron en profundidad estructuras sociales básicas. No es este el momento de discutir si dichos cambios supusieron una superación de la modernidad o una post- 
modernidad (como han mantenido autores como Lyotard, Baudrillard, Derrida, Jameson, etc.) o una acentuación de la propia modernidad, una modernidad tardía (Giddens, 1993), sino simplemente de reflejar los cambios acaecidos en la esfera de la seguridad y el riesgo.

Las causas de este cambio habría que situarlas sobre todo en la confluencia de tres factores: (a) El proceso de individualización creciente de la sociedad, que tiene como consecuencia la ampliación del margen de libertad y el aumento de las opciones vitales del individuo, a lo que habría que añadir la erosión de estructuras sociales como la familia (Lipovetsky, 2002 y 2003). (b) El debilitamiento de las solidaridades colectivas como garantía frente a los riesgos que ahora presentan consecuencias más agudas. (c) La desmitificación de la ciencia y la técnica como ahuyentadoras de riesgos, ofreciendo ellas mismas otros nuevos, como por ejemplo la amenaza ecológica. A todos estos factores habría que sumar el gran catalizador de todos los riesgos en la sociedad postmoderna: la globalización, proceso que conecta y multiplica los posibles riesgos locales. El caso es que, como consecuencia de estos hechos, en la esfera de la seguridad se produjeron modificaciones en el paradigma vigente. Así, se extinguió o amortiguó la creencia en el progreso (Campillo, 1985); y se produjo una crisis de legitimación en las principales instituciones sociales, entre ellas la ciencia. Las sociedades se hicieron más flexibles, más fluidas, estando el papel de los individuos sujeto a un mayor número de modificaciones a lo largo de la vida.

Podemos afirmar, como hemos visto en el primer y segundo epígrafe, que nos encontramos en un periodo de transición entre el modelo de seguridad total propio de la modernidad y un modelo de seguridad parcial propio de la post-modernidad o modernidad tardía. Es curioso señalar que todo periodo de cambio genera ansiedad social y respuestas potencialmente violentas. Todos estos factores hacen que nos demos cuenta en la actualidad de que el control de los riesgos es siempre limitado. El ser humano en estas sociedades vuelve a ser consciente de los límites del control sobre la naturaleza y el propio ser humano.

Nos encontramos, por tanto, lejos de sociedad donde se conocía al vecino y se sabía lo que era posible esperar. Vivimos en una sociedad donde priman los intereses individuales sobre los grupales y eso nos desconcierta (cada uno esconde sus cartas debido a la gran competitividad) y nos sentimos cada vez más amenazados por las personas que nos rodean (desconocidos). Echamos en falta la seguridad, una cualidad crucial para la vida feliz del hombre moderno, pero una cualidad que la sociedad en la que habitamos es incapaz de ofrecer.

A todo esto se suma el hecho de que las fuentes de inseguridad no son visibles, no podemos localizarlas con precisión. La inseguridad nos afecta a todos, inmersos como estamos en un mundo fluido e impredecible de desregularización, flexibilidad, competitividad e incertidumbre endémicas, pero cada uno de nosotros sufre la ansiedad individualmente, como un problema privado. Ante este hecho 
tendemos a buscar un remedio para el malestar que la inseguridad nos provoca. Esto se refleja en la mayor preocupación por la seguridad de nuestro entorno: el hogar, el vecindario o las propiedades. El otro comienza a ser sospechoso, portador de lo imprevisto e imprevisible. Los extraños son la amenaza encarnada. Amenaza, por lo demás, invisible e ilocalizable.

El carácter incontrolable de los riesgos en la sociedad postmoderna ha sido remarcado por los más destacados sociólogos (Beck, 2002; Beck, Giddens y Lash, 1997), que han acuñado el término "sociedad del riesgo" para describirlo. El mismo entorno global en el que nos movemos no hace sino aumentar la incertidumbre. Colectivos que antes tenían fuertes mecanismos sociales para protegerse de los riesgos, se ven ahora incapaces de frenar los nuevos riesgos (Kirby, 2002 y 2005). La incapacidad de los Estado para frenar los coletazos más dañinos de la globalización para sus propias poblaciones (deslocalizaciones productivas, riesgos medioambientales, etc.), el debilitamiento de los mecanismos de aseguramiento colectivo propios de la sociedad civil (iglesias, familias y toda una pléyade de organizaciones), aumentan la vulnerabilidad de colectivos que antes no se sentían en situación de riesgo. Amplias capas de la población, las otrora opulentas clases medias, sienten que su situación es crecientemente más vulnerable. El riesgo se ha asentado entre nosotros y es parte consustancial de las sociedades postmodernas.

\section{EL INDIVIDUO EN LA SOCIEDAD INSEGURA}

Algunos autores se han fijado especialmente en la ansiedad social que produce este nuevo entorno (Hollway y Jefferson, 1997; Wilkinson, 2001). La ansiedad social, como un estado agitación, inquietud y angustia del ánimo, deriva directamente de esta nueva situación de vulnerabilidad percibida o real. En este caso, resulta escasamente relevante la distinción entre vulnerabilidad real y vulnerabilidad percibida, entre riesgos reales y riesgos percibidos. Aplicando el conocido Teorema de Thomas, los riesgos percibidos, aún sin serlos realmente, tienen consecuencias reales en el comportamiento de los individuos. Si los individuos perciben, como así parece, que este mundo es más peligroso y entraña nuevos y crecientes riesgos se sentirán, sin duda, vulnerables. En todo caso, el individuo se siente más vulnerable debido a la multiplicación de riesgos debidos a la globalización: des-localizaciones productivas, pérdida de derechos laborales, nuevas redes mafiosas internacionales, terrorismo transnacional, etc.

El sujeto es consciente, en mayor o menor grado, de los riesgos del mundo global y de su incapacidad de afrontarlos como individuo. Se siente vulnerable y, en buena lógica, esta percepción conduce a un continuo estado de ansiedad. Además, como afirma Keneth Gergen, la conciencia postmoderna "se nos presenta como una pizarra en blanco donde los sujetos pueden inscribir su identidad, borrarla y 
volver a escribirla, en la medida en que se lo permita o los inste a ello una red incoherente de relaciones personales en permanente expansión y cambio" (1992: 287). Las seguridades ontológicas de la modernidad quedan atrás y el individuo debe hacer y rehacer continuamente sus relaciones y su autoconciencia, lo que contribuye continuamente a situarle en una cuerda floja existencial. La inseguridad, en consecuencia, constituye un elemento vertebrador de la conciencia individual postmoderna.

El estado de ansiedad social generado por esa nueva forma de conciencia produce dos tipos de respuestas. En primer lugar, se habla de un retorno a las comunidades primarias defensivas frente a la sociedad red surgida de la globalización: "Como los nuevos procesos de dominación a los que reacciona la gente están insertos en los flujos de información, la construcción de la familia y la comunidad ha de basarse en la inversión de estos flujos. Dios, patria, familia y comunidad proporcionan códigos eternos e indestructibles en torno a los cuales se organizará una contraofensiva" (Castells, 1999, vol. 2: 89). Los individuos buscan la seguridad perdida en instituciones primarias como la familia, la nación o la religión. La disolución en estas "comunidades orgánicas" proporciona la individuo la seguridad perdida y le permite superar el estado de ansiedad. Asimismo, el debilitamiento del Estado como gran mecanismo protector, en su vertiente militar-orden internacional, económica y social -Estado de Bienestar- (Anderson, Brook y Cochrane,1995); influye, sin duda, en un sentimiento de desamparo que conlleva la búsqueda de nuevas fuentes de seguridad.

En segundo, la desaparición de un entorno estable sin duda genera fenómenos de ansiedad, que pueden llevar a una frustración que, ocasionalmente, puede estallar en manifestaciones violentas. Aunque no existe una relación directa entre la frustración y la violencia, como bien ha demostrado la psicología social al mantener que no existe equivalencia absoluta entre un contenido mental y una conducta efectiva (Buceta, 1992: 102-109); en no pocas ocasiones, la frustración puede generar violencia contra la parte que no ha cumplido su papel dentro de las expectativas sociales generadas. Es lógico pensar que la comprensión significativa del mundo por parte de los actores tendrá un correlato en su conducta. Si los ciudadanos de los países occidentales han sido socializados en la creencia de ser ciudadanos iguales ante la ley -con derecho a un acceso igualitario a los bienes materiales y simbólicos de la sociedad-, protegidos por las instituciones en los aspectos básicos de su existencia; obviamente, se sentirán defraudados y frustrados ante el incumplimiento de sus expectativas básicas sobre el funcionamiento social. Tanto los recientes disturbios en la periferia de las grandes ciudades francesas como los anteriores en Los Ángeles, pueden muy bien ser una muestra del incumplimiento de estas expectativas básicas.

Ambas respuestas no son contradictorias, sino que más bien se retroalimentan. Una respuesta violenta producida por unas expectativas frustradas 
y una creciente sensación de vulnerabilidad tiene una gran repercusión, amplificada por los medios de comunicación de masas (Gil Calvo, 2004), que puede revertir en un recogerse en el seno de comunidades primarias de los individuos que se sienten amenazados por esta violencia. Y, por el contrario, los grupos defensivos pueden actuar violentamente contra otros en defensa de sus micro-espacios de seguridad. La ansiedad social genera un modelo convivencial basado en la percepción de un riesgo endémico, en el cual la única respuesta es la de considerar al "otro" un potencial enemigo -y viceversa-, y la búsqueda de un orden hobbesiano. Podemos citar, entre otras, como muestras de estas respuestas contradictorias: (a) La creación de un "negocio" en torno a la seguridad. Las compañías dedicadas a la seguridad privada, la video-vigilancia, el procesamiento de datos, etc., no hacen sino crecer y aumentar sus beneficios. (b) Se están creando una serie de "entornos seguros" donde se refugian las capas altas de la sociedad. Estos "guetos" se encuentran vigilados por compañías privadas las 24 horas durante los 365 días del año. Se está produciendo una proliferación del hábitat suburbano, de la urbanización cerrada y blindada. Y (c) la desaparición de la calle, como territorio inseguro, frente al centro comercial, vigilado continuamente y dotado de toda una serie de comodidades.

La seguridad es una de las prioridades de los ciudadanos en todos los países avanzados, que tiene como contrapartida la exigencia a las autoridades de mayores medidas de seguridad (mayor control policial, asistencia, etc.). Cada vez se tolera menos la imprevisión, siendo las catástrofes naturales y los fenómenos climatológicos responsabilidad de los políticos y de los gobiernos, que deben prever y tratar de minimizar sus efectos. La necesidad de seguridad desborda incluso el ámbito estrictamente nacional y lleva a una demanda de una seguridad "colectiva internacional". Para ello se recurre a los ejércitos, considerando las grandes potencias (tanto la Unión Europea como los Estados Unidos) que éstos son más una fuerza policial internacional que una fuerza de conquista al modo clásico.

Es curioso señalar, en este orden de cosas, que los debates actuales en torno a la seguridad recogen una vieja polémica. Cuando el primer ministro británico Tony Blair afirma que los ciudadanos deben elegir entre libertad y seguridad, no está sino recogiendo el pensamiento del sociólogo decimonónico británico Herbert Spencer. Para Spencer, la libertad y la seguridad están interconectadas: un mayor grado de libertad implica uno menor de seguridad y, al contrario, un menor grado de libertad conlleva un mayor grado de seguridad. La decisión sobre el grado de seguridad, por tanto, es enteramente política. Habremos de decidir en qué tipo de sociedad queremos vivir y qué límites fijamos. En este campo colisionan la concepción moderna de la seguridad, con la idea de progreso firmemente arraigada en la población, y la situación postmoderna en la que la globalización implica el anclaje de una incertidumbre endémica en el seno de nuestras sociedades. Por ello, la gestión del riesgo se encuentra con la paradoja de individuos que reclaman amplias cuotas de libertad y, al tiempo, mínimos niveles de incertidumbre. 


\section{CONCLUSIONES}

El artículo ha tratado de presentar las principales dimensiones de uno de los fenómenos más relevantes del devenir social actual: la inseguridad colectiva. La globalización ha modificado profundamente las convenciones sociales más arraigadas en torno a la seguridad. Los Estados-Nación trataron de crear una red que asegurara a sus ciudadanos de los principales riesgos presentes en sus vidas. Sin embargo, la creciente interconexión mundial impide la aplicación de estos principios de aseguramiento. La inseguridad colectiva es ubicua y los mecanismos tradicionales de control de riesgo fallan. Los individuos sufren estos cambios globales y solamente pueden dar respuestas individuales que, obviamente, fallan, pues no pueden proporcionar un escudo eficaz ante los nuevos riesgos mundializados.

La concepción de la seguridad ha variado ampliamente en el transcurso de la historia. Desde el fatalismo propio de las sociedades tradicionales, se ha llegado a la confianza del mundo moderno que comienza a ser socavada en las sociedades postmodernas. Fruto de este continuado cambio social, la colectividad social se ve atrapada, sin desearlo, en una paradoja: se cree y se necesita un mundo donde existan mecanismos que aseguren la existencia; $y$, sin embargo, el mundo globalizado debilita la creencia en el progreso y en la ciencia como base del aseguramiento colectivo, al tiempo que la interdependencia global auspicia nuevos riegos e imposibilita un control de los riesgos. Aparecen nuevos colectivos vulnerables que son incapaces de hacer frente a estos nuevos riesgos.

En definitiva, la inseguridad endémica parece uno de los aspectos más relevantes del nuevo orden social propiciado por la globalización económica, política, social y cultural. La cultura postmoderna incluye la inseguridad como una de sus dimensiones básicas y, en consecuencia, los individuos estructuran su yo social en respuesta a las demandas de un entorno percibido como crecientemente hostil. La inseguridad subjetiva es parte ya de la conciencia colectiva postmoderna. La seguridad es plenamente un elemento constitutivo del debate social y político, al estar enraizada en un nuevo tipo de conciencia individual propia de nuestro tiempo. Como afirma Eduardo Sabrovksy: "La inseguridad es un estado peculiar de la conciencia contemporánea, una inquietud difusa y persistente, un «malestar de la cultura»..., que no se puede explicar de modo meramente empírico” (1996: 55). La inseguridad parece haberse asentado, pues, como una dimensión ontológica de nuestras sociedades, y no meramente como un fenómeno transitorio. 


\section{BIBLIOGRAFÍA}

ANDERSON, J., BROOK, C. y COCHRANE, A., (Eds.) (1995): A Global World? Re-Ordering Political Space, The Open University-Oxford University Press, Oxford.

BAUMAN, Z., (2003): Comunidad. En busca de seguridad en un mundo hostil, Siglo XXI, Madrid.

- (2002): La sociedad sitiada, FCE, México.

BECK, U., (2003): Sobre el terrorismo y la guerra, Paidós, Barcelona.

- (2002), La sociedad del riesgo global, Siglo XXI, Madrid.

BECK, U., GIDDENS, A. y LASH, S., (1997): Modernización reflexiva. Política, tradición y estética en el orden social moderno, Alianza, Madrid.

BERICAT ALASTUEY, E., (Dir.) (2003): El conflicto cultural en España. Acuerdos y desacuerdos entre los españoles, CIS, Madrid.

BUCETA FACORRO, L., (1992): Fundamentos psicosociales de la información, Editorial Centro de Estudios Ramón Areces, Madrid.

BURY, J., (1971): La idea del progreso, Alianza, Madrid.

CAMPILlO, A., (1985): Adiós al progreso. Una meditación sobre la Historia, Anagrama, Barcelona.

CASTELLS, M., (1999): La era de la información, Alianza, Madrid, 3 vols.

DA AGRA, C. et. al. (Eds.) (2003): La seguridad en la sociedad del riesgo: un debate abierto, Atelier, Barcelona.

DOUGLAS, M., (1996): La aceptabilidad del riesgo según las ciencias sociales, Paidós, Barcelona.

ERICSON, R.V. y HAGGERTY, K.D., (1997): Policing the Risk Society, Claredon Press, Oxford.

GARCÍA MORENTE, M., (1980): Ensayos sobre el progreso, Dorcas, Madrid. GERGEN, K., (1992): El yo saturado, Paidós, Barcelona.

GIDDENS, A., (1993): Consecuencias de la modernidad, Alianza, Madrid.

- (2000): Un mundo desbocado. Los efectos de la globalización en nuestras vidas, Taurus, Madrid.

GIL CALVO, E., (2004): El miedo es el mensaje, Alianza, Madrid.

HOLLWAY, W. y JEFFERSON, T., (1997): "The risk society in an age of anxiety: Situating the fear of crime", en British Journal of Sociology, 48 (2): 255-266.

HOLZMANN, R., SHERBURNE-BENZ, L. y TESLIUC, E., (2003): Social Risk Management: The World Bank Approach to Social Protection in a Globalizing World, World Bank, New York.

KIRBY, P., (2002): "The World Bank or Polanyi: Markets, Poverty and Social Well-being in Latin America", en New Political Economy, 7(2): 199-219.

- (2005): "El impacto social de la globalización: De la pobreza a la vulnerabilidad", Working Paper inédito presentado en la Universidad Rey Juan Carlos: 43 pp. 
LINDSEY, N.K., (2004): Communication and Responsibility in the "Risk Society", University of London, London.

LIPOVETSKY, G., (1992): La era del vacio, Anagrama, Barcelona.

- (1993): Metamorfosis de la cultura liberal, Anagrama, Barcelona.

LÓPEZ CEREZO, J.A. y LUJÁN LÓPEZ, J.L., (2000): Ciencia y política del riesgo, Alianza, Madrid.

LUJÁN, J.L., (2005): Gobernar los riesgos. Ciencia y valores en la sociedad del riesgo, Biblioteca Nueva, Madrid.

MÉNDEZ, P., (2002): "Sobre el concepto de <<riesgo>>”, en Nómadas. Revista Crítica de Ciencias Sociales y Jurídicas, 5. [En línea: http://redalyc.uaemex.mx/ redalyc/pdf/181/18100507.pdf].

NISBET, R., (1980): Historia de la idea de progreso, Gedisa, Barcelona.

RITZER, G., (1999): La McDonalización de la sociedad. Un análisis de la racionalización de la vida cotidiana, Ariel, Barcelona.

ROMEO CASABONA, C.M., (2005): Conducta peligrosa e imprudencia en la sociedad del riesgo, Comares, Granada.

SABROVKSY, E., (1996): El desánimo. Ensayo sobre la condición contemporánea, Nobel, Oviedo.

SENNETT, R., (2003): El respeto. Sobre la dignidad del hombre en un mundo de desigualdad, Anagrama, Madrid.

SLOVIC, P., (2000): The Perception of Risk, Earthscan Publications, London.

VV.AA. (1993): Número monográfico: ¿Hacia una sociedad del riesgo?, en Revista de Occidente, 150.

WILKINSON, I., (2001): Anxiety in a Risk Society, Routledge, London.

WOLTON, D., (2004): La otra mundialización. Los desafios de la cohabitación cultural global, Gedisa, Barcelona.

KALDOR, M., (2003): La sociedad civil global. Una respuesta a la guerra, Tusquets, Barcelona. 